\title{
A Result Analysis of Localization of License Plate Number Using Dynamic Image Processing Techniques and Genetic Algorithms
}

\author{
Bharti Tiwari ${ }^{1}$, Pravin Tiwari ${ }^{2}$ \\ M.Tech Scholar, Department of ECE, Takshila Institute of Engineering and Technology, Jabalpur, India ${ }^{1}$ \\ Professor, Department of ECE, Takshila Institute of Engineering and Technology, Jabalpur, India ${ }^{2}$
}

\begin{abstract}
In this research analysis, a design of a new genetic algorithm (GA) is introduced to detect the locations of the License Plate (LP) symbols. In this paper open ALPR (Automated license plate Recognition) method is used to observe candidate objects within the image. This methodology depends on distinctive the key characteristic of a number plate the concentration of characters with robust light-on-dark edges.
\end{abstract}

Keywords: Genetic algorithms, image processing, image representations, license plate detection, machine vision, road vehicle identification, sorting crossover.

\section{INTRODUCTION}

This License Plate Recognition (LPR) plays an important role in numerous applications such as unattended parking lots [1], security control of restricted areas [5], and traffic safety enforcement [8], [9]. This task is quite challenging due to the diversity of plate formats and the non-uniform outdoor illumination conditions during image acquisition, such as backgrounds [2], [10], illumination vehicle speeds and distance ranges between the camera and the vehicle. Therefore, most approaches work only under restricted conditions such as fixed illumination, limited vehicle speed, designated routes, and stationary backgrounds.

A typical system for LPR (License Plate Recognition) consists of four parts, i.e., obtaining an image of the vehicle, license plate localization and segmentation, character segmentation and standardization, and character recognition. The performance of the locating operation is crucial for the entire system, because it directly influences the accuracy and efficiency of the subsequent steps. However, it is also a difficult obstacle to overcome because of different illumination conditions and various complex backgrounds. In this project proposed many methods of locating the license plates, such as the edge detection method line sensitive filters to extract the plate areas, the window method and the mathematics morphology method. Although these algorithms can process the license plates location, they possess formidable disadvantages such as sensitivity to brightness, longer processing time, and lack of versatility in adapting to the varying environment. Research in and were based on the intensity distribution in the plate's space with respect to its neighbourhood wherever the plate is considered as Maximally Stable External Region (MSER). The applied detection algorithms ranged from windowbased statistical matching ways to extremely intelligentbased technique that used neural networks or fuzzy logic.
GAs has been used seldom due to their high process needs. In GA (genetic algorithm) was used to search for the simplest mounted rectangular area having a similar texture options as that of the prototype template. In GA (genetic algorithm) was wont to locate the plate vertically when detection the left and right limits based on horizontal symmetry of the vertical texture bar graph round the plate's space. GA (genetic algorithm) was used in to recognize the LP (License Plate) symbols to not detect the LP. Another cluster of researchers tried to control the problem from the texture perspective to differentiate between text and different image varieties.

\section{THEORY}

As way as extraction of the plate region is concerned, techniques primarily based upon combinations of edge statistics and mathematical morphology [3]-[6] featured excellent results. In these ways, gradient magnitude and their local variance in a picture are computed. they're based on the property that the brightness modification within the license plate region is additional remarkable and additional frequent than otherwise. Block-based processing is additionally supported [7].

Then, regions with a high edge magnitude and high edge variance are known as possible license plate regions. Since this technique doesn't depend upon the edge of vehicle plate boundary, it may be applied to a picture with unclear license plate boundary and may be implemented simply and quick. a drawback is that edge-based ways alone will hardly be applied to complicated pictures, since they're too sensitive to unwanted edges, which can additionally show high edge magnitude or variance (e.g., the radiator region within the front read of the vehicle). In spite of this, once combined with morphological steps that eliminate 
Vol. 4, Issue 1, January 2017

unwanted edges within the processed pictures, the license plate extraction rate is relatively high and fast compared to different ways.

\section{III.METHOD}

There were many techniques used to achieve the plate recognition. The plate recognition procedures in general are performed in the following steps:

\section{A. Location of the registration plate}

The When the vehicle is in range of the video camera, the vehicle triggers an inductive loop detector so that the camera captures an image of the front of the vehicle. The digital image will be stored in the memory ready for image processing. A segmenting algorithm on the full image will be used for segmenting the car image, and the segmented part that matches the registration plate features will be extracted for the optical character recognition.

\section{B. Normalizing of the registration plate area}

When the registration plate has been located, it could have a tilted orientation and/or not be of the standard size. This could prevent the features of the plate and its characters from matching the expected features.

Thus it is most important to have the extracted plate normalized. This will be achieved when the system measures the tilt slope and the heights of the plate or the characters and, with an appropriate technique, apply a correction to normalize the image.

C. Character feature extraction:

Segmentation is required to separate each character in the registration plate image. This is also achieved by segmentation being applied to the normalized registration plate area in order to segment the characters from the plate background.

D. Characters identification:

The identification of characters IS only necessary for the systems that use an identification matching technique for image recognition. During this stage, the system produces a unique identification for every character used in the registration plate.

\section{E. Preparing the database:}

The database consists of the registration plate character templates or identifications, where these templates or identifications are produced from actual registration plates.

F. Character recognition:

A full image feature or an identification profile of the extracted character will be compared to the database for matching according to the best correlation factors.

In the proposed algorithm to be developed will locate strong edges which are spaced proportionally given the expected size of the plate relative to the overall image. A strong edge is defined as a vertical line where adjacent pixels have high luminance deltas, relative to other areas of the scene. Using which the accuracy of license plate detection will be high analysis. This technique has the advantage of analysing texture in an unlimited number of directions and scales. A method for license plate location based on the Gabor transform is presented. The results were encouraging once applied to digital pictures acquired strictly during a fixed and specific angle, however the strategy is computationally expensive and slow for pictures with large analysis. For a two-dimensional (2-D) input image of size $\mathrm{N} \times \mathrm{N}$ and a 2-D Gabor filter of size $\mathrm{W}$ $\times \mathrm{W}$, the procedure complexity of 2-D Gabor filtering is within the order of $\mathrm{W} 2 \mathrm{~N} 2$, providing the image orientation is fixed at a particular angle. Therefore, this methodology was tested on small sample pictures and it was reported that additional work remains to be done in order to alleviate the limitations of 2-D Gabor filtering. Genetic programming (GP) and genetic algorithms (GAs) were additionally implemented for the task of license plate location. gp is sometimes much more computationally intensive than the GAs, though the 2 evolutionary paradigms share a similar basic algorithmic rule.

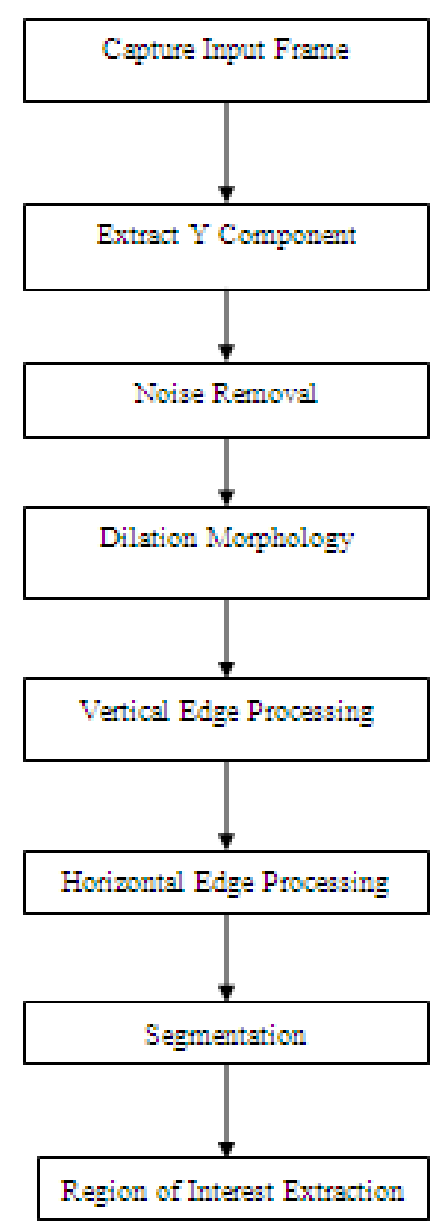

Fig.1 Flowchart showing license plate detection algorithm

\section{IV.RESULT}

The proposed system has been implemented using MATLAB. 


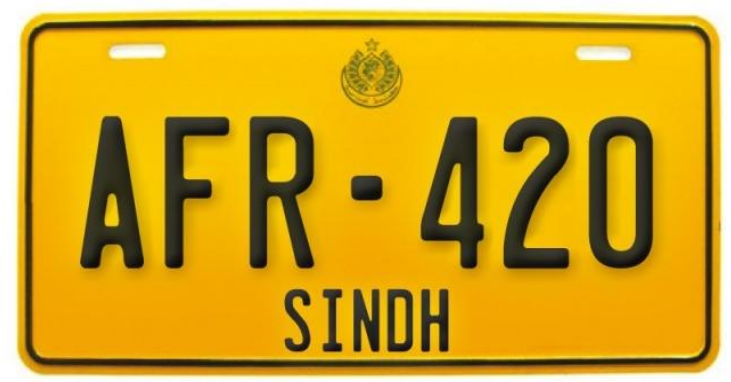

Fig.2 Main image

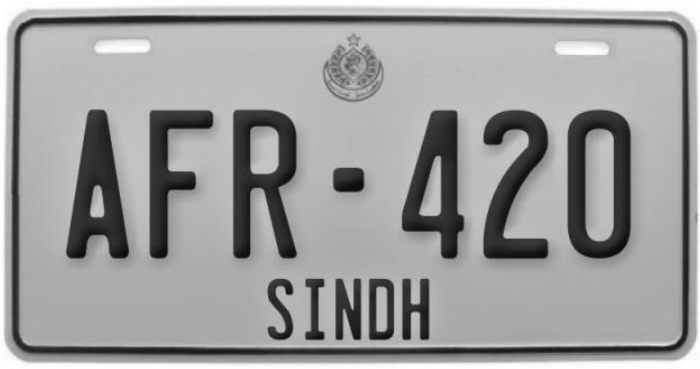

Fig.3 Gray image

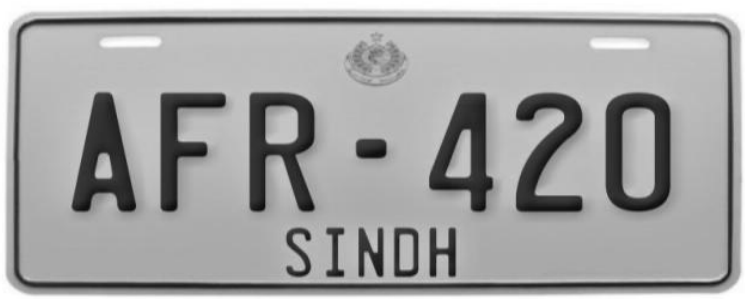

Fig.4 Dilated image

\section{AFR- 420 SINDH}

Fig.5 Gray image

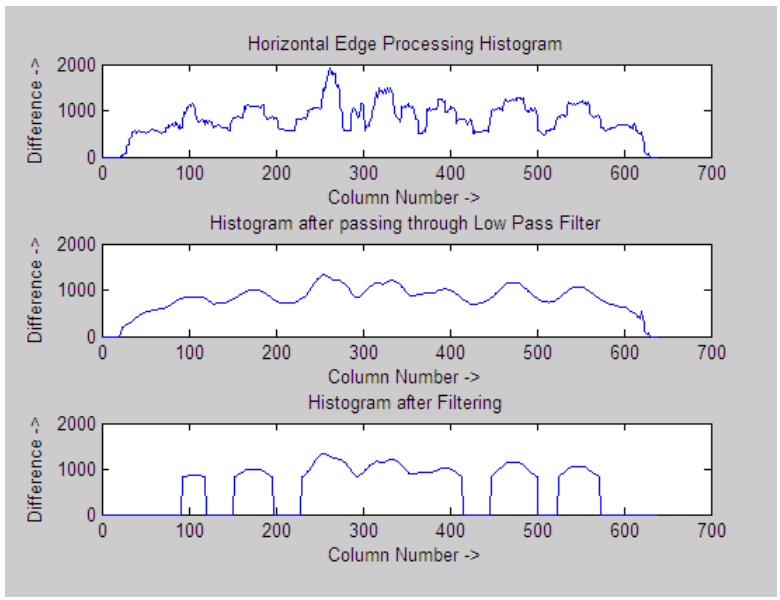

Fig.6 Horizontal edge processing histogram

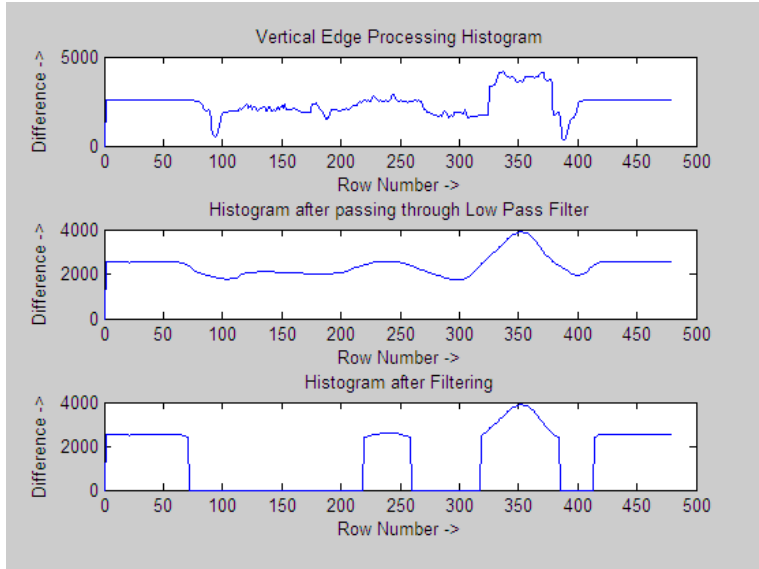

Fig.7 Vertical edge processing histogram

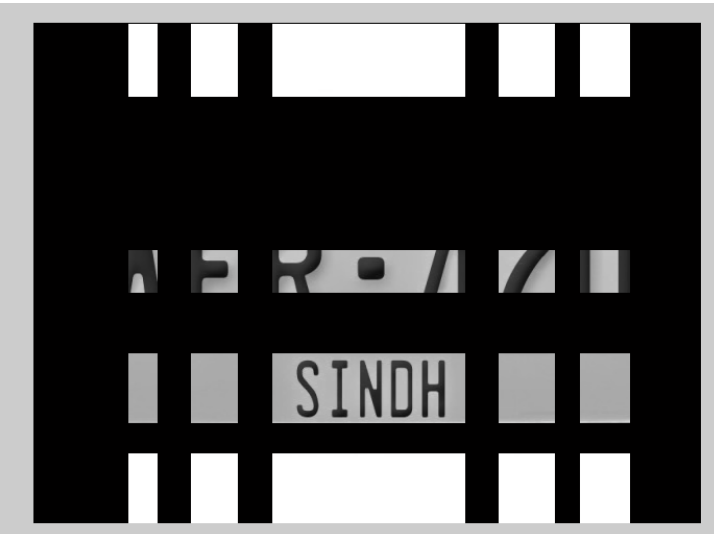

Fig. 8 Histogram image after filtering

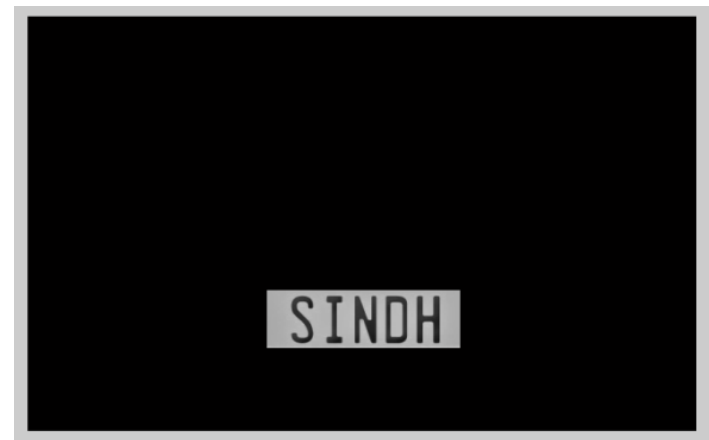

Fig.9 Output image

\section{V.CONCLUSION}

The In this paper proposed many different techniques for plate recognition. In this project the results were encouraging and a new approach for solving the LP detection problem relying only on the geometrical layout of the LP symbols has been experimentally proved. In this the proposed system possessed high immunity to changes in illumination either temporarily or spatially through the plate area.

\section{REFERENCES}

[1] G. Abo samra, f. Khalefah "localization of license plate number using dynamic image processing techniques and genetic 
algorithms" IEEE tran. On evol. Computing vol. 18 no. 2 pp. 1-14 2014

[2] D.-J. Kang "Dynamic programming-based method for extraction of license plate numbers of speeding vehicles on the highway" International Journal of Automotive Technology, Vol. 10, No. 2, pp. 205-210 2009.

[3] S. Chang, L. Chen, Y. Chung, and S. Chen, "Automatic License Plate Recognition" IEEE Tran. on Inte.Trans. Sys., vol. 5, no. 1, pp.42-53 2004 .

[4] C.E.Anagnostopoulos, V. Loumos, Ioannis E. Anagnostopoulos, and Eleftherios Kayafas, "A License Plate-Recognition Algorithm for Intelligent Transportation System Applications" IEEE Trans. on Intell. Transp. Sys., vol. 7, no. 3, pp. 377-392 2006.

[5] Clemens Arth, Florian Limberger, Horst Bischof "Real-Time License Plate Recognition on an Embedded DSP-Platform" Graz University of Technology Institute for Computer Graphics and Vision, Austria.

[6] Halina Kwaśnicka and Bartosz Wawrzyniak "License plate localization and recognition in camera pictures", Gliwice, Poland November 13-15, 2002.

[7] M. Deriche, " GCC License Plates Detection and Recognition Using Morphological Filtering and Neural Networks," Int J. on Comp. Sci. and Info Security, IJCSIS, vol. 8, No. 8, pp. 263-269, Dec, 2010.

[8] O. Villegas, D. Balderrama, H. Domínguez and V. Sánchez," License Plate Recognition Using a Novel Fuzzy Multilayer Neural Network," International Journal of Computers", Issue 1,vol. 3, 2009.

[9] S.H. Mohades Kasaei, S.M. Mohades Kasaei and S.A. Monadjemi, "A Novel Morphological Method for Detection and Recognition of Vehicle License Plate," American Journal of Applied Science, vol.6 no. 12, pp. 2066-2070, 2009.

[10] A. Theja, S. Jain, A. Aggarwal and V. Kandanvli, "License Plate Extraction Using Adaptive Threshold and Line Grouping," International Conference on Signal Processing Systems (ICSPS), vol.1, no., pp. 211-214, 5-7 July 2010 